\title{
¿Ayudamos cuando la situación de necesidad es abstracta? El efecto prosocial de la disposición a incluir el macro-ambiente en el espacio vital
}

\author{
Sergio Salgado ${ }^{1 *}$, Carolina González-Suhr ${ }^{2}$ y Luis Oceja ${ }^{2}$ \\ ${ }^{1}$ Departamento de Administración y Economía, Universidad de la Frontera, Chile \\ ${ }^{2}$ Departamento de Psicología Social y Metodología, Universidad Autónoma de Madrid, España
}

\begin{abstract}
Resumen: En este trabajo se analiza el efecto prosocial de la inclusión del macro-ambiente en el espacio vital. Es decir, se propone que la medida en que las personas perciben que su vida se desarrolla en contextos ambientales amplios (p.ej., la sociedad y el mundo) facilita la conducta prosocial cuando la situación de necesidad se presenta de modo abstracto (p.ej., muy amplia y lejana). Para comprobar esta hipótesis se realizaron dos estudios. En el Estudio 1 se construyó y analizó un instrumento de medida del grado en que se incluye el ambiente macro en el espacio vital (EV-macro). En el Estudio 2, se incluyó una manipulación del tipo de situación de necesidad (abstracta vs. concreta). Los resultados mostraron que el EV-macro se relacionó con una mayor conducta prosocial cuando la necesidad fue planteada en términos relativamente más abstractos.

Palabras clave: Espacio vital; Ambiente macro; Conducta prosocial; Necesidad abstracta.
\end{abstract}

\section{Introducción}

Es evidente que los seres humanos realizamos acciones que tienen consecuencias positivas para quienes nos rodean. En Psicología Social a este tipo de acciones se las ha agrupado bajo el término genérico de conducta prosocial y su explicación ha sido abordada desde enfoques muy diversos (para una revisión ver Dovidio, Piliavin, Schroeder y Penner, 2006).

Naturalmente, la mayor parte de este tipo de acciones las realizamos en nuestro entorno más próximo, beneficiando a personas con quien mantenemos una relación real y actual (p.ej., la familia, los amigos o un colectivo al cual pertenecemos). No obstante, el ser humano también busca mejorar el bienestar de individuos que no forman parte de su vida cotidiana. Una explicación plausible a este tipo de comportamiento es la conocida hipótesis empatía-altruismo (Batson, 1991; 2011). Este proceso puede explicar la conducta prosocial, tanto si se trata de una persona en necesidad (p. ej., Batson, Lishner, Cook y Sawyer, 2005) como si implica a más de un individuo, porque si bien en un principio la empatía surge hacia un otro, en ciertas ocasiones su efecto puede extenderse también a otras personas cercanas a él (Batson y Ahmad, 2009). Desde una perspectiva diferente, otra variable que influye en la conducta de ayuda es el sentimiento de oneness (Cialdini, Brown, Lewis, Luce y Neuberg, 1997) que deriva del proceso de fusión con el otro (self-other merging). Ahora bien, el ser humano parece ir incluso más allá y en ciertas ocasiones busca la mejora de situaciones que afectan a individuos que le son completos desconocidos (p. ej., un número indeterminado de niños de otro continente). Sin embargo,

* Dirección para correspondencia [Correspondence address]: Sergio Salgado. Departamento de Administración y Economía, Universidad de la Frontera, Casilla 54 D, Temuco, Chile.

E-mail: sergiosalgados@yahoo.es
Title: Do we help when the situation of need is abstract? The prosocial effect of the disposition to including the macro-environment in life space. Abstract: We analyze the prosocial effect of including the macroenvironment in life space. Namely, it is proposed that the extent to which people perceive that their life takes place in broader environmental contexts (e.g., the society, the world) will facilitate prosocial behavior when the situation of need is presented in an abstract fashion (e.g., very broad and distant). Two studies were conducted to test this hypothesis. In Study 1, we created and tested an instrument to measure the extent to which the macro-environment is included in life space (EV-macro). In Study 2 was included a manipulation of the kind of the need situation (abstract vs. concrete). Results showed that the EV-macro was related to prosocial behavior when the need was presented in relatively more abstract terms.

Key words: Life space, Macro-environment, Prosocial behavior, Abstract need.

si la situación de necesidad implica cierto nivel de abstracción - ya sea por su lejanía, amplitud o complejidad- entonces el efecto de la empatía o del oneness puede ser menor. Una forma de solucionar esta disminución consiste en aumentar la similaridad entre el actor y la víctima mediante, por ejemplo, un perfil de personalidad congruente (Krebs, 1975), una misma religión (Dovidio y Morris, 1975), o parecido racial (Gaertner y Dovidio, 1977). Pero, ¿existe otro proceso que pueda aumentar la conducta prosocial cuando la situación de necesidad es abstracta y la similaridad es poco probable?

Incluir el macro-ambiente en el espacio vital como antecedente de conducta prosocial

Desde un punto de vista lewiniano el espacio vital -o situación total- es definido como persona más ambiente psicológico. El ambiente psicológico implica los elementos del ambiente que la persona realmente percibe. A su vez, el distinto grado de inclusión de estos elementos implica una mayor o menor preponderancia en el campo de fuerzas que afecta a la persona y su comportamiento (Lewin, 1946/1997).

A pesar de la importancia que tiene el concepto de espacio vital para la Psicología Social, el modo en que éste se configura no ha sido abordado directamente en la búsqueda de explicaciones para la conducta prosocial. En este trabajo nos centramos en uno de los aspectos relevantes de dicha configuración: la inclusión psicológica del nivel ambiental macro, y analizamos su relación con uno de los mayores retos sociales actuales: la promoción de conducta prosocial hacia víctimas en situaciones de necesidad abstractas, ya sea por su lejanía, complejidad o gran magnitud.

Tomando como punto de partida el planteamiento de Magnusson y Allen (1983; citado por López-Soler, 1996) el espacio vital puede analizarse en función de dos parámetros: 
amplitud y proximidad. El primero, en línea con el modelo ecológico de Bronfenbrenner $(1977,1979)$ se refiere a la amplitud de los ambientes percibidos, es decir, al continuo que comprende los niveles ambientales micro, meso y macro. Los niveles micro y meso hacen referencia a los contextos más concretos y acotados, propios de la vida cotidiana (p. ej., la familia); y el nivel macro alude a los contextos vitales más amplios y abstractos (p.ej., la sociedad) ${ }^{1}$. El segundo parámetro se refiere al grado en que la persona percibe como próximo cada uno de estos niveles, es decir, en qué medida considera que su acción se desarrolla en los contextos micro, meso y macro (Jessor, 1981; Pervin, 1978).

En línea con lo anterior, planteamos la siguiente hipótesis: la presentación de una situación de necesidad en términos abstractos (p. ej. que un número indeterminado de niños de otro continente no tengan acceso a la educación) facilitará la conducta de ayuda en aquellas personas que perciben como próximos los contextos más amplios (p. ej. la ciudad, la sociedad o el mundo); es decir, aquellos que incluyen el nivel macro en su espacio vital.

Para comprobar esta hipótesis se llevaron a cabo dos estudios. En el Estudio 1 se desarrolló una medida del grado en que se incluye el ambiente macro en el espacio vital (en adelante, EV-macro) y en el Estudio 2 se comprobó que la presentación de una situación de necesidad en términos abstractos o concretos modera la relación de esta medida con la conducta prosocial.

\section{Estudio 1}

El objetivo de este primer estudio fue desarrollar una medida del EV-macro. En línea con nuestra propuesta, para representar la dimensión ambiental macro se utilizaron conceptos relativos a contextos amplios y abstractos (i.e., ciudad, sociedad y mundo) y para representar la dimensión ambiental micro y meso se utilizaron conceptos relativos a contextos más acotados y concretos (i.e., familia, amigos y trabajo). Como puede apreciarse en la Figura 1, el instrumento evalúa el grado en que se perciben como próximos cada uno de estos seis contextos presentándolos en orden creciente de amplitud y abstracción. Por lo tanto, se esperaba contrastar que el EV-macro, medido a través de este nuevo instrumento, corresponde a una dimensión factorial diferenciada (H1).

Por otra parte, con el fin de contrastar la validez convergente del instrumento, se incluyó el cuestionario de valores de Schwartz (SVS; Schwartz, 1992, 1994). En una investigación previa (Schwartz, Sagiv y Boehnke, 2000) se comprobó que las personas que puntúan más alto en los valores del cuadrante Autotrascendencia sienten una mayor preocupación por problemáticas de nivel macro (p. ej., "Gente en el mundo muere de hambre"). Por lo tanto, esperamos que el

\footnotetext{
${ }^{1}$ Definiciones similares ya se han utilizado en investigaciones previas (Boehnke, Schwartz, Stromberg y Sagiv, 1998; Schwartz, Sagiv y Boehnke, 2000) para referirse a preocupaciones de nivel macro ("acerca de la sociedad, el mundo e incluso el universo") y micro ("acerca de uno mismo y sus extensiones" o "del endogrupo o extensiones del yo").
}

EV-macro muestre una relación positiva y significativa con el cuadrante Autotrascendencia de la SVS 2 (H2).

\begin{tabular}{|c|c|c|c|c|c|c|c|c|c|c|}
\hline $\begin{array}{l}\text { ¿En qué medida crees que tu vida se desarrolla } \\
\text { en los siguientes ambientes?: }\end{array}$ & \multicolumn{2}{|c|}{ Nada } & \multicolumn{3}{|c|}{ Algo } & \multicolumn{3}{|c|}{ Bastante } & \multicolumn{2}{|c|}{ Totalmente } \\
\hline a) En familia & 1 & 2 & 3 & 4 & 5 & 6 & 7 & 8 & 9 & 10 \\
\hline b) Con amigos & 1 & 2 & 3 & 4 & 5 & 6 & 7 & 8 & 9 & 10 \\
\hline c) En el trabajo & 1 & 2 & 3 & 4 & 5 & 6 & 7 & 8 & 9 & 10 \\
\hline d) En tu ciudad & 1 & 2 & 3 & 4 & 5 & 6 & 7 & 8 & 9 & 10 \\
\hline e) En la sociedad & 1 & 2 & 3 & 4 & 5 & 6 & 7 & 8 & 9 & 10 \\
\hline f) En el mundo & 1 & 2 & 3 & 4 & 5 & 6 & 7 & 8 & 9 & 10 \\
\hline
\end{tabular}

Figura 1. Instrumento de medida del grado en que se incluye el ambiente macro en el espacio vital (EV-macro).

Por último, teniendo en cuenta que el hecho de presentarse como alguien que se desarrolla en ambientes vitales amplios (p. ej., en el mundo) podría ser considerado como una forma de obtener aprobación social y, que la búsqueda de esta aprobación podrían afectar las puntuaciones a nuestro instrumento, incluimos una medida de deseabilidad social (Strahan y Gerbasi, 1972) para controlar la influencia de dicha variable.

\section{Método}

\section{Participantes}

Ciento cuarenta y nueve estudiantes de primer año de Psicología de la Universidad Autónoma de Madrid participaron en el estudio como parte de unas prácticas de formación (21 hombres y 128 mujeres; Edad media $=19.66, D t=4.89)$. El universo de estudiantes de Psicología de la UAM puede considerarse socioeconómicamente heterogéneo.

\section{Instrumentos}

Incluir el macro-ambiente en el espacio vital (EV-macro). Para medir el EV-macro se preguntó a los participantes “¿En qué medida crees que tu vida se desarrolla en los siguientes ambientes?" y a continuación se presentaron seis contextos (en familia, con los amigos, en el trabajo, en tu ciudad, en la sociedad, en el mundo) cada uno de los cuales fue evaluado mediante una escala de nueve puntos $(1=\mathrm{Nada}, 4=$ Algo, 7 $=$ Bastante, $10=$ Totalmente).

Perfil de valores (SVS). Para medir el perfil de valores se utilizó el cuestionario desarrollado por Schwartz (1992, 1994) y adaptado al español por Ros y Schwartz (1995). Esta versión presenta un total de 60 valores expresados a través de un concepto seguido por una breve frase explicativa ( $p$.

\footnotetext{
2 Schwartz $(1992,1994)$ ha desarrollado un modelo global de valores que se organiza en una estructura cuasi-circunfleja que representa el patrón total de las mutuas relaciones de conflicto y congruencia. Dicha estructura se resume en dos dimensiones ortogonales: a) Automejora vs. Autotrascendencia, que se refiere al grado en que los valores enfatizan, o en la búsqueda del autointerés, o en la preocupación por el bienestar de los demás; y b) Abierto al Cambio/Conservación, que se refiere al grado en que los valores hacen hincapié, o en la búsqueda de la libertad de acción, independencia y nuevas experiencias, o en la auto-restricción y la resistencia al cambio.
} 
ej., Igualdad: Igualdad de oportunidades para todos). Para completarlo se pide al participante que evalúe cada valor según "la importancia que tiene para ti como principio guía en tu vida" utilizando una escala de 9 puntos $(-1=$ opuesto a mis valores, $1=$ nada importante, $3=$ importante, $5=$ muy importante, $7=$ de suprema importancia).

Deseabilidad Social. Se utilizó la traducción al español de la escala Marlowe-Crowne en su versión abreviada desarrollada por Strahan y Gerbasi (1972). Esta medida identifica la tendencia a responder buscando la aprobación social. Para ello al participante se le pide indicar en qué medida se identifica con diez afirmaciones (p. ej., "Nunca nadie me ha caído excesivamente mal") en formato de respuesta Verdadero/Falso.

\section{Procedimiento}

El investigador entregó un cuadernillo con los instrumentos a estudiantes que accedieron a participar voluntariamente como parte de su formación académica. La batería de cuestionarios fue completado en treinta minutos aproximadamente. Todos los participantes firmaron un formulario de consentimiento donde se explicó el objeto del estudio y la confidencialidad de los datos personales aportados.

\section{Resultados}

En primer lugar, se analizaron los estadísticos descriptivos del instrumento. Las puntuaciones obtenidas mostraron una amplitud de respuesta media y desviación típica adecuadas. Las puntuaciones más altas fueron obtenidas por los ambientes micro/meso (amigos, familia y trabajo; $M \mathrm{~s}=$ $7.84,7.14$ y $6.42 ; D t s=1.50,1.83$ y 1.94 , respectivamente) mientras que, los ambientes macro mostraron puntuaciones relativamente más bajas (sociedad, ciudad y mundo; $M \mathrm{~s}=$ 6.28, 6.09 y $5.58 ; D t \mathrm{~s}=2.04,2.02$ y 2.53 , respectivamente) lo cual muestra que los participantes percibieron que su acción suele desarrollarse en los niveles micro y meso.

En segundo lugar, se exploró la estructura del modelo mediante un Análisis Factorial Exploratorio (AFE) utilizando el programa FACTOR (Lorenzo-Seva y Ferrando, 2006). Debido a que este programa no admite valores perdidos, seis puntuaciones en blanco fueron reemplazadas por la media de su dimensión teórica, y dos participantes fueron descartados por dejar más de un ítem sin contestar. Luego, considerando que los ítems fueron evaluados por medio de una escala politómica, el AFE fue calculado a partir de la matriz de covarianzas policóricas (Olsson ,1979a, 1979b) con el método de extracción MRFA (Minimum Rank Factor Analysis; ten Berge y Kiers, 1991) y rotación Promax normalizada. Previamente, para determinar el número de factores que debían conservarse se llevó a cabo un Análisis paralelo (Horn, 1965). Este análisis mostró que los dos primeros factores presentaron autovalores muestrales mayores, tanto a la media de autovalores aleatorios como al percentil 95 de los mismos. A partir de este resultado se configuró el AFE, extrayéndose dos factores que explicaron en conjunto un
$88.12 \%$ de la varianza (matriz reducida). En el primer factor, que explicó un 55.04\% de la varianza, saturaron los ítems de la dimensión macro (ciudad, sociedad y mundo) con pesos factoriales iguales a $.37, .63$ y .55, respectivamente. En el segundo factor, que explicó un $33.08 \%$ de la varianza, saturaron los ítems micro/meso (familia, amigos y trabajo) con pesos factoriales iguales a $.33, .32$ y .57 , respectivamente. Esta solución factorial mostró un buen nivel ajuste; RMSR (Root Mean Square for Residuals) $=.02$ (valores menores .08 pueden considerarse de buen ajuste; p. ej., Harman, 1962). A continuación, se construyó el índice EV-macro por medio de la suma ponderada de los ítems de este factor, el cual mostró una consistencia interna adecuada (Alpha de Cronbach [en adelante, $a]=.77)$. Utilizando este índice se obtuvo el patrón de correlaciones con los valores de la SVS. De acuerdo a lo esperado, el índice EV-macro mostró una correlación muy significativa con el cuadrante Autotrascendencia, $r$ (142) $=$ .25 ; $p<.01$; en especial con el valor Universalismo y en menor medida con el valor Benevolencia, $r$ s $(143)=.23$ y .18 ; ps $<.01$ y .05 , respectivamente. Este índice también se relacionó con el cuadrante Apertura al cambio, $r(141)=.21 ; p$ $<$.05. Por último, se comprobó que el índice EV-macro no se relacionó con la medida de deseabilidad social, $r(144)=$ $.10 ; p>.20$.

Análisis complementarios. De modo análogo, decidimos explorar el mismo patrón de correlaciones con el índice EVmicro/meso $(a=.33)$. Los resultados mostraron un patrón claramente diferente de los relacionados con la variable de este estudio (EV-macro) y coherente con nuestro planteamiento. Concretamente, este índice EV-micro/meso se relacionó sólo con el cuadrante Conservación de la SVS, $r$ (138) $=.32 ; p<.01$, en especial con los valores Seguridad y Conformidad, $r \mathrm{~s}(138,140)=.30$ y .27 , respectivamente, $p \mathrm{~s}<$ .01 ; y en menor medida con Tradición, $r(138)=.14 ; p<.10$. Además, este índice sí se mostró relacionado con la medida de deseabilidad social, $r(140)=.22 ; p<.01$. Ahora bien, dado el bajo nivel de consistencia interna, estos resultados deben considerarse como provisionales.

\section{Discusión}

El principal objetivo del Estudio 1 fue construir y poner a prueba un instrumento de medida del EV-macro. Para construir la medida de esta dimensión del espacio vital se utilizaron conceptos relativos a contextos amplios y abstractos (i.e., ciudad, sociedad y mundo) mientras que, la dimensión micro/meso fue construida utilizando conceptos relativos a contextos más acotados y concretos (i.e., familia, amigos, trabajo). El modelo fue contrastado por medio de un análisis factorial exploratorio el cual reveló una solución consistente con la propuesta.

Por otra parte, para contrastar la validez convergente del instrumento se analizó su relación con el cuestionario de valores de Schwartz. En concreto, basados principalmente en un trabajo previo (Schwartz, Sagiv y Boehnke, 2000) se estableció que el EV-macro se relacionaría sólo con el cuadrante 
Autotrascendencia de la SVS y los resultados presentados así lo confirmaron. También se pudo constatar que el índice EV-macro no se mostró relacionado con la variable deseabilidad social (Strahan y Gerbasi, 1972) lo cual permite descartar la influencia de la búsqueda de aprobación social en las puntuaciones a nuestra medida.

A la luz de estos resultados se realizó un nuevo estudio, cuyo objetivo fue contrastar la hipótesis principal de nuestro planteamiento: que la presentación de una situación de necesidad en términos abstractos facilitará la conducta de ayuda en aquellas personas que incluyen en mayor medida el nivel macro en su espacio vital.

\section{Estudio 2}

El Estudio 2 tuvo como fin comprobar que el EV-macro se relaciona positiva y significativamente con la conducta prosocial (H1) especialmente cuando la situación de necesidad es presentada en términos abstractos (H2). Para ello, una nueva muestra respondió al instrumento desarrollado en el Estudio $1 \mathrm{y}$, dos semanas más tarde, a la mitad de estos participantes se les presentó una situación de necesidad en términos relativamente más abstractos y a la otra mitad en términos relativamente más concretos.

Asimismo, también se esperaba que la mayor inclusión del nivel macro en el espacio vital facilite la generación de objetivos de naturaleza relativamente más abstracta cuando la situación de necesidad es presentada en términos más abstractos (H3). Para comprobarlo se midió el grado en que la persona atribuye su decisión de ayudar a dos tipos de fin: uno relativamente más abstracto (i.e., ser justo ${ }^{3}$ ) y uno relativamente más concreto (i.e., ayudar al individuo).

\section{Método}

\section{Participantes}

Setenta estudiantes de primer año de Psicología de la Universidad Autónoma de Madrid participaron en el estudio (10 hombres y 60 mujeres; Edad media $=20.88 ; D t=7.15)$. Los participantes se apuntaron voluntariamente en distintas prácticas que implicaban la asistencia a dos actividades diferentes. El procedimiento que aquí se presenta (Estudio 2) fue presentado como "Introducción a la evaluación de aspectos sociales".

\section{Procedimiento}

El estudio se realizó en dos etapas. En la primera, los participantes completaron el instrumento descrito y analizado en el Estudio 1 de forma individual. En la segunda, reali-

\footnotetext{
${ }^{3}$ Se tiene en consideración que el principio de justicia ha sido consistentemente relacionado con un razonamiento prosocial más abstracto, independiente, e incluso potencialmente conflictivo con el fin de mejorar el bienestar de un individuo en concreto (p. ej., Batson, 1995; Batson, Klein, Highberger y Shaw, 1995; Oceja, 2008)
}

zada dos semanas más tarde, se llevó a cabo la situación experimental. Estas actividades fueron presentadas como procedimientos independientes. En cada una se solicitó la edad, el género y una clave de participante, lo cual permitió relacionar los datos obtenidos.

La situación experimental se llevó a cabo en el laboratorio de cabinas de la Facultad de Psicología de la UAM. Estas cabinas son espacios individuales e insonorizados que contienen un escritorio, una silla y un ordenador. Los participantes fueron recibidos por una investigadora que explicó el propósito de la actividad: evaluar una serie de anuncios publicitarios que algunas organizaciones estaban pilotando. Además, con el fin de obtener la mayor implicación posible por parte de cada participante, indicó que cada anuncio sería evaluado sólo por una persona y, por lo tanto, que de sus evaluaciones dependería que los anuncios fuesen mejorados o modificados. También se informó que el procedimiento se realizaría de forma individual, siguiendo las instrucciones a través de una presentación confeccionada en Power Point. A continuación, los participantes entraron en la cabina. En primer lugar, leyeron y firmaron el formulario de consentimiento de participar, en el que se explicó el carácter confidencial de los datos personales recogidos. Las cabinas de investigación cuentan con una pequeña ventana en la puerta, a espaldas del participante, de modo que mientras se realizó el procedimiento la investigadora pudo verificar que se seguía el orden requerido.

La presentación contenía siete diapositivas. En la primera se mencionó nuevamente el propósito del estudio. La segunda diapositiva mostró una carta en la que el Sr. José Tesero, Director de la Fundación Calidad de Vida, agradecía la participación y explicaba que la labor de esta ONG internacional era mejorar la vida de niños que viven en situaciones difíciles. En cuanto al anuncio que sería evaluado, señalaba que había sido diseñado con el fin de incrementar su presencia en los medios (ambos, el Sr. Tesero y la fundación, son ficticios).

Manipulación de la necesidad: concreta vs. abstracta. En la tercera diapositiva -justo antes de presentar el anuncio- se dio la siguiente instrucción: "A continuación te presentaremos el caso de Guddi. Por favor, mientras lees el anuncio piensa en Guddi como [una niña]/[una de las muchas niñas] que se encuentra en esta situación". Como puede observarse entre corchetes, a la mitad de los participantes se le presentó una situación de necesidad relativamente más concreta (i.e., una niña en necesidad), mientras que a la otra mitad se le presentó una situación de necesidad relativamente más abstracta (i.e., una de las muchas niñas en necesidad).

El anuncio. En la cuarta diapositiva se presentó el anuncio, que incluyó un texto y una fotografía. En el texto se explicó la situación de Guddi, una niña de 12 años natural de Sierra Leona que vive en condiciones de pobreza extrema, sin acceso a los servicios de agua potable, salud y educación. En la fotografía se mostró una imagen de Guddi.

Conducta prosocial. A continuación (quinta diapositiva) se presentó una carta de la directora de la investigación, la Dra. 
Sariego, en la que se daba la oportunidad de colaborar con la fundación: "Hemos pensado que algunos participantes podrían estar interesados en ayudar en la situación descrita en el anuncio. Por lo tanto, sugerimos a la organización que lo diseñó que diera esta opción por si alguien lo deseaba. Si estás interesado en tener más información acerca de esta oportunidad, por favor abre el Sobre 2, si no estás interesado sigue adelante con la tarea. En todo caso, ten en cuenta que tu participación no te obliga de ningún modo a aceptar este ofrecimiento".

Los participantes que decidieron abrir el Sobre 2 encontraron un formulario donde se explicó que podían ayudar dedicando parte de su tiempo a distintas tareas, tales como empaquetar medicinas o alimentos. Si el participante decidía ayudar debía rellenar el formulario aportando sus datos de contacto (nombre y correo electrónico) y señalar cuánto tiempo de colaboración deseaba comprometer. Para indicar este tiempo se proporcionó una escala con los siguientes rangos: 10 a 30 minutos, 30 minutos a 1 hora, 1 a 2 horas, de 2 a 3 horas, de 3 a 4 horas, de 4 a 5 horas, más de 5 horas. En este formulario se recordó, una vez más, que la participación en el estudio no obligaba en ningún modo a aceptar esta posibilidad, en cuyo caso bastaba con dejar el formulario en blanco.

Activación de objetivos. En la sexta diapositiva, se pidió contestar a un cuestionario que incluyó dos ítems cuyo objetivo era medir hasta qué punto los participantes atribuían que su decisión se relacionaba con el hecho de "ayudar a Guddi" (fin concreto), o "ser justo" (fin abstracto). Estos ítems fueron evaluados utilizando una escala de 7 puntos $(1=$ nada, 7 $=$ totalmente).

Entrevista post-experimental. En la séptima diapositiva se indicó el fin de esta actividad y que diera aviso a la investigadora. Entonces se realizó una breve entrevista para aclarar posibles dudas o sospechas. Ninguno de los participantes presentó sospechas sobre la autenticidad del anuncio o de la ayuda solicitada. Finalmente, se agradeció y despidió al participante.

\section{Resultados}

Inclusión del ambiente macro en el espacio vital (EV-macro). En primer lugar, se analizó la estructura del EV-macro completado dos semanas antes de la situación experimentalpor medio de un SEM (Amos 18; Máxima verosimilitud). El tamaño de la muestra resulta ajustado y suficiente considerando la regla básica de un mínimo de 10 observaciones por cada ítem (p. ej., Evaritt, 1975), o la regla de al menos 5 observaciones por cada parámetro a estimar (p. ej., Hair, Black, Babin, Anderson y Tatham, 2006). El modelo incluyó dos variables latentes (EV-macro y EV-micro/meso) cuya covarianza no se restringió y seis variables observadas; tres por cada variable latente según la definición del modelo. Los resultados de este análisis mostraron buenos niveles de ajuste $\left(x^{2}=4.59[p=.80] ; x^{2} / \mathrm{Gl}=.57 ; \quad C F I=1.0 ;\right.$ TLI $=1.0 ;$
RMSE $A=.00)^{4}$. A continuación, se procedió a calcular los índices correspondientes a cada una de estas dimensiones: $\mathrm{EV}$-macro $(a=.88)$ y EV-micro/meso $(a=.30)$.

Tipo de situación de necesidad (concreta vs. abstracta) y conducta prosocial. Un total de 44 de los 70 participantes $(62,9 \%)$ decidieron ayudar. Aunque la manipulación "concreta vs. abstracta" no tuvo un efecto significativo en la conducta de ayuda, sí mostró una tendencia en línea con la investigación previa que muestra que, por lo general, se ayuda en mayor medida cuando la situación de necesidad es más concreta (Small y Loewenstein, 2003; Kogut y Ritov, 2005). En este sentido, 25 de los 35 participantes $(71,4 \%)$ decidieron ayudar cuando la situación de necesidad fue presentada de modo relativamente más concreto, y 19 de los 35 participantes $(54,3 \%)$ cuando la situación se presentó de modo relativamente más abstracto; $\chi^{2}=2.20 ; p=.21$ (exacta), $p=$ .13(asintótica) .

EV-macro y conducta prosocial. Como se puede observar en la Tabla 1, el índice EV-macro mostró una correlación significativa con la cantidad de ayuda comprometida sólo en la condición de necesidad abstracta $(p s<.05)$. Además, entre aquellos que decidieron ayudar, el índice EV-macro mostró una correlación significativa con el grado en que atribuyeron dicha decisión al fin más abstracto de seguir un principio de justicia $(p<.05)$.

Tabla 1. Correlaciones de Pearson de la inclusión de los ambientes macro y micro/meso con la decisión de ayudar, la cantidad de ayuda y el tipo de objetivo activado para cada situación de necesidad.

\begin{tabular}{llccc}
\multirow{2}{*}{ Necesidad } & & $\begin{array}{c}\text { Cantidad } \\
\text { de ayuda }\end{array}$ & $\begin{array}{c}\text { Fin } \\
\text { abstracto }\end{array}$ & $\begin{array}{c}\text { Fin } \\
\text { concreto }\end{array}$ \\
\hline \multirow{4}{*}{ Abstracta } & EV-macro & $n=35$ & $n=19$ (sí ayudan) \\
\cline { 2 - 5 } Concreta & EV-micro/meso & .12 & $.51^{*}$ & .31 \\
& & $n=35$ & $n=25$ (sí ayudan) \\
\cline { 2 - 5 } & EV-macro & -.11 & $-.37 \dagger$ & -.07 \\
& EV-micro/meso & .18 & .21 & $.53^{* *}$ \\
\hline
\end{tabular}

A continuación, analizamos el efecto moderador de la manipulación, es decir, del modo en que se presentó la necesidad (abstracta vs. concreta) en el efecto prosocial del EVmacro (i.e., en la cantidad de ayuda comprometida). Para ello, el modelo en estudio fue representado en un diagrama de rutas (Amos 18): dos variables observadas cuya covarianza no se restringió (EV-macro y EV-micro/meso) predicen la variable dependiente observada: la cantidad de ayuda ofrecida. En línea con lo esperado, el ratio crítico del efecto de la moderación (Critical Ratios Differences between Parameters) reveló una diferencia cuasi significativa en los pesos de regresión del EV-macro sobre la cantidad de ayuda: $\beta$ s $=.25$ y -.14 para la situación planteada de forma más abstracta y más concreta, respectivamente; $z=1.91, p<.06$. Mientras que, la diferencia de los pesos de regresión del EV-micro/meso sobre esta misma variable se dio en dirección opuesta, aunque

${ }^{4} x^{2}=$ Chi cuadrado; $d f=$ Grados de libertad $; x^{2} / d f=$ Chi cuadrado relativo; $C F I=$ Índice de ajuste comparativo de Bentler; TLI $=$ CoeficienteTuckerLewis; RMSE $A=$ Error cuadrático medio de aproximación. 
no llegó a ser significativa: $\beta \mathrm{s}=-.03$ y .33 para las situaciones abstracta y concreta, respectivamente; $z=0.98, p>.32$.

Adicionalmente, se realizó el mismo análisis para verificar si este efecto moderador también se presentó en la atribución del tipo de objetivo perseguido. El resultado mostró un efecto significativo en la diferencia de los pesos de regresión del EV-macro sobre la atribución de perseguir un objetivo más abstracto (ser justo): $\beta \mathrm{s}=.35$ y -.29 cuando la situación fue planteada de forma más abstracta y más concreta, respectivamente; $z=2.27, p<.03$. Por el contrario, la diferencia en los pesos de regresión del EV-micro/meso sobre esta variable se mostró en sentido inverso, aunque en este caso tampoco llegó a ser significativa: $\beta \mathrm{s}=-.19$ y .46 cuando la situación fue planteada de forma más abstracta y más concreta, respectivamente; $z=1.24, p>.21$.

\section{Discusión}

Los resultados del Estudio 2 confirmaron la moderación hipotetizada: plantear la situación en términos más abstractos promocionó la intervención de aquellas personas que perciben que su acción se desarrolla en contextos amplios, es decir, aquellos que incluyen el ambiente macro en su espacio vital. Asimismo, presentar la necesidad en estos términos también moderó la relación entre la inclusión del ambiente macro y la atribución del comportamiento de ayuda como resultado de la activación de un objetivo (ser justo) coherente con nuestra hipótesis. En cambio, plantear la situación de necesidad en términos más particulares y concretos bloqueó tanto el efecto prosocial de la inclusión del macro-ambiente, como también la atribución de este comportamiento a la persecución de un objetivo abstracto.

\section{Discusión final}

Existe una gran variedad de procesos que aumentan y reducen la probabilidad de que en un momento dado y en un contexto concreto una determinada persona inicie una conducta que beneficia a otra u otras personas. Algunos de estos procesos son activados o bloqueados por las circunstancias que rodean la decisión en ese momento y contexto, y otros procesos están más relacionados con las disposiciones de la persona que toma la decisión. Reconociendo esta unidad de análisis Persona X Situación, en el presente trabajo hemos enfocado el concepto de espacio vital (Lewin, 1946/1997; Bronfenbrenner, 1977, 1979) para proponer una vía distinta relacionada con la generación de conducta prosocial: la inclusión del nivel ambiental macro en el espacio vital.

Nosotros planteamos que esta inclusión aumenta la probabilidad de que se realice una mayor conducta prosocial siempre y cuando se den unas determinadas circunstancias coherentes con dicho proceso.

Para poner a prueba este planteamiento se realizaron dos estudios. En el Estudio 1 se obtuvo un instrumento de medida de la inclusión del ambiente macro en el espacio vital (i.e., EV-macro). En el Estudio 2 se comprobó la hipótesis de que el EV-macro se relaciona con la conducta prosocial cuando la situación de necesidad se plantea en términos más abstractos. Esto tiene consecuencias tanto a nivel teórico como práctico.

A nivel teórico las principales consecuencias son dos. La primera es que en este trabajo se ha tomado como punto de partida la configuración del espacio vital, un enfoque no abordado previamente que abre un amplio abanico de investigación sobre las variaciones y mejoras en el instrumento para medirlo, la forma de presentar la situación de necesidad y la relación de estos procesos con otros procesos relacionados con la conducta prosocial (p.ej., altruismo, identificación grupal). La segunda es que la inclusión psicológica del ambiente macro sugiere ser una vía distinta para la promoción de conducta prosocial que ha demostrado ser especialmente eficaz en un tipo de situación (i.e., abstracta) donde el poder explicativo de otros procesos (p. ej., empatía, oneness, similaridad) tiende a verse mermado. Este resultado abre un amplio rango de consecuencias prácticas. Por ejemplo, existen miles de organizaciones alrededor del mundo preocupadas por encontrar fórmulas más eficientes, tanto para concienciar y encontrar nuevo apoyo, como también para mantener el apoyo de quienes ya colaboran en la tarea de solucionar situaciones de necesidad que por su magnitud, complejidad o lejanía pueden ser definidos como problemas abstractos. Consideramos que los resultados de este trabajo pueden arrojar alguna luz sobre cómo actuar en este tipo de situaciones.

\section{Limitaciones y futuras investigaciones}

Teniendo en cuenta la investigación previa, en el presente trabajo hemos asumido que la situación de necesidad presentada, crítica en la comprobación de nuestro modelo, era relativamente abstracta, y que los elementos relacionados con la generación de empatía, oneness o similaridad eran más débiles o percibidos en menor medida. En futuras investigaciones sería conveniente medir o, mejor aún, manipular los escenarios de conducta con el fin de establecer más claramente: (a) cuáles son las características que facilitan que una situación se perciba como abstracta, (b) en qué caso el efecto de la inclusión del ambiente macro en el espacio vital ofrece mejores resultados respecto de la realización de conducta prosocial, (c) en qué medida las variables de empatía, oneness y similaridad podrían ser complementarias al proceso aquí propuesto, y (d) en qué situaciones, en cambio, podría surgir un conflicto que afectara negativamente a la promoción de la conducta prosocial.

Agradecimientos.- Este trabajo ha contado con el patrocinio del Programa Capital Humano Avanzado (Conicyt, Chile) y del Ministerio de Educación de España (PSI2011-28720). Además, queremos expresar nuestro mayor agradecimiento por su ayuda en la realización de este trabajo a Tamara Ambrona y Belén López-Pérez. 


\section{Referencias}

Batson, C. D. (1991). The altruism question: Toward a social-psychological answer. Hillsdale, NJ: Erlbaum Associates.

Batson, C. D. (1995). Prosocial Motivation: Why do we help others? En A. Tesser (Ed.), Advanced Social Psychology (p.p. 333-381). New York: McGraw-Hill.

Batson, C. D. (2011). Altruism in Humans. New York: Oxford University Press.

Batson, C. D. y Ahmad, N. Y. (2009). Using empathy to improve intergroup attitudes and relations. Social Issues and Policy Review, 3, 141.

Batson, C. D., Klein, T. R., Highberger, L. y Shaw, L. L. (1995). Immorality from empathy-induced altruism: When compassion and justice conflict. Journal of Personality and Social Psychology, 68, 1042-1054.

Batson, C. D., Lishner, D. A., Cook, J. y Sawyer, S. (2005). Similarity and nurturance: Two possible sources of empathy for strangers. Basic and Applied Social Psychology, 27, 15-25.

Boehnke, K., Schwartz, S. H., Stromberg, C., Sagiv, L. (1998). The structure and dynamics of worry: Theory, measurement, and cross-cultural replications. Journal of Personality, 66, 745-782.

Bronfenbrenner, U. (1977). Lewinian space and ecological substance. Journal of Social Issues, 33, 199-212.

Bronfenbrenner, U. (1979). The ecology of buman development. Experiment by nature and design. Cambridge, MA: Harvard University Press.

Cialdini, R. B., Brown, S. L., Lewis, B. P., Luce, C. y Neuberg, S. L. (1997). Reinterpreting the empathy-altruism relationship: When one into one equals oneness. Journal of Personality and Social Psychology, 73, 481-494.

Dovidio, J. F. y Morris, W. N. (1975). Effects of stress and commonality of fate on helping behavior. Journal of Personality and Social Psychology, 59, 249-260

Dovidio, J., Piliavin, J., Schroeder, D. y Penner, L. (2006). The social psychology of prosocial behavior. Mahwah, NJ: Lawrence Erlbaum.

Everitt, B. S. (1975). Multivariate analysis: the need for data and other problems. British Journal of Psychiatry, 126, 237-240.

Gaertner, S. L. y Dovidio, J. E. (1977). The subtlety of with racism, arousal, and helping behavior. Journal of Personality and Social Psychology, 35, 691707.

Hair, J. F., Black, W. C., Babin, B. J., Anderson, R. E. y Tatham, R. L. (2006). Multivariate Data Analysis. New Jersey: Pearson Prentice Hall.

Harman, H. H. (1962). Modern Factor Analysis (Segunda Edición). University of Chicago Press, Chicago.

Horn, J. L. (1965). A rationale and test for the number of factors in factor analysis. Psychometrika, 30, 179-185.

Hornstein, H. A. (1972). Promotive tension: The basis of prosocial behavior from a Lewinian perspective. Journal of Social Issues, 28, 191-218.

Jessor, R. (1981). The perceived environment in psychological theory and research. En D. Magnusson (Ed.), Toward a psychology of situations: An interactional perspective (pp. 297-317). New York: Lawrence Erlbaum Associates.
Kogut, T. y Ritov, I. (2005). The "Identified Victim" effect: An identified group, or just a single individual? Journal of Behavioral Decision Making, 18, 157-167.

Krebs, D. (1975). Empathy and altruism. Journal of Personality and Social Psychology, 32, 996-1003.

Kogut, T. y Ritov, I. (2005b). The singularity effect of identified victims in separate and joint evaluations. Organizational Behavior and Human Decision Processes, 97, 106-116.

Lewin, K. (1946/1997). Behavior and development as a function of the total situation. En G.W. Lewin y D. Cartwright (Eds.), Resolving social conflicts y Field theory in social science (pp. 337-381). Washington, DC: American Psychological Association.

López-Soler, C. (1996). Interacción Persona-Situación. En A. Fierro (Coordinador), Manual de Psicología de la Personalidad (pp. 407-435). Barcelona: Editorial Paidós.

Lorenzo-Seva, U. y Ferrando, P.J. (2006). FACTOR: A computer program to fit the exploratory factor analysis model. Behavioral Research Methods, Instruments and Computers, 38, 88-91.

Magnusson, D. y Allen, V. (1983). Human development and Interactional perspective. New York: Academic Press.

Oceja, L.V. (2008). Overcoming empathy-induced partiality: Two rules of thumb. Basic and Applied Social Psychology, 30, 176-182.

Olsson, U. (1979a). Maximum likelihood estimation of the polychoric correlation coefficient. Psychometrika, 44, 443-460.

Olsson, U. (1979b). On the robustness of factor analysis against crude classification of the observations. Multivariate Behavioral Research, 14, 485500 .

Pervin, L. A. (1978). Current Controversies and Issues in Personality. New York: Wiley.

Ros, M. y Schwartz, S. (1995). Jerarquía de valores de la Europa Occidental: una comparación transcultural. Revista Española de Investigaciones Sociológicas, 69, 69-88.

Schwartz, S. H. (1992). Universals in the content and structure of values: Theoretical advances and empirical test in 20 countries. En M. P. Zanna (Ed.), Advances in Experimental Social Psychology, Vol. 25 (pp. 1-65). New York: Academic Press.

Schwartz, S. H. (1994). Are there universal aspects in the structure and contents of human value? Journal of Social Issues, 50, 19-45.

Schwartz, S. H., Sagiv, L., Boehnke, K. (2000). Worries and Values. Journal of Personality, 68, 309-346. DOI: 10.1111/1467-6494.00099.

Small, D. A. y Loewenstein, G. (2003). Helping a victim or helping the victim: Altruism and identifiability. Journal of Risk and Uncertainty, 26, 5-16.

Ten Berge, J.M.F. y Kiers, H.A.L. (1991). A numerical approach to the exact and the approximate minimum rank of a covariance matrix. Psychometrika, 56, 309-315.

(Articulo recibido: 14-09-2011, revisado: 25-04-2012, aceptado: 25-04-2012) 